\title{
Bandwidth and repetition rate programmable Nyquist sinc-shaped pulse train source based on intensity modulators and four-wave mixing
}

\author{
S. Cordette, ${ }^{*}$ A. Vedadi, M. A. Shoaie, and C.-S. Brès \\ Ecole Polytechnique Fédérale de Lausanne (EPFL), Photonic Systems Laboratory (PHOSL), STI-IEL, \\ Station 11, CH-1015 Lausanne, Switzerland \\ *Corresponding author: steevy.cordette@epfl.ch
}

Received August 25, 2014; revised October 22, 2014; accepted October 27, 2014; posted October 27, 2014 (Doc. ID 221304); published November 24, 2014

\begin{abstract}
We propose and experimentally demonstrate an all-optical Nyquist sinc-shaped pulse train source based on intensity modulation and four-wave mixing. The proposed scheme allows for the tunability of the bandwidth and the full flexibility of the repetition rate in the limit of the electronic bandwidth of the modulators used through the flexible synthesis of rectangular frequency combs. Bandwidth up to $360 \mathrm{GHz}$ at $40 \mathrm{GHz}$ rate and up to 45 frequency lines at $5 \mathrm{GHz}$ rate are demonstrated with $40 \mathrm{GHz}$ modulators. (c) 2014 Optical Society of America

OCIS codes: (060.0060) Fiber optics and optical communications; (060.2330) Fiber optics communications; (190.4380) Nonlinear optics, four-wave mixing; (320.5540) Pulse shaping.

http://dx.doi.org/10.1364/OL.39.006668
\end{abstract}

With the ever-growing demand for telecommunication bandwidth, highly efficient spectral techniques are currently thoroughly investigated in order to optimize the capacity of fiber optics networks. Two digital techniques are commonly used: orthogonal-frequency division multiplexing (OFDM), where a superchannel composed of sincshaped subcarriers is generated, and Nyquist-WDM where the data symbols are carried by Nyquist-shaped pulses in the time domain [1]. Recently, the concept of orthogonal time-division multiplexing (O-TDM) of optically generated Nyquist pulses was demonstrated [2]. In this scheme, the ultra-short Nyquist pulses are generated all-optically and time multiplexed with no intersymbol interference (ISI), taking advantage of the orthogonality of Nyquist pulses. Various techniques to generate optical Nyquist pulses have been demonstrated including spectral reshaping of mode-locked laser [2] or fiber optical parametric amplification using one degenerated pump []ㅡ or two dissimilar frequency pumps in order to achieve uniform pulse generation over a wide bandwidth [4]. These techniques allow for the generation of Nyquist pulses of a few picoseconds duration down to sub-picosecond. However, reducing the complexity of these schemes to a viable solution is not straightforward. Moreover, the generated Nyquist pulses exhibit a non-rectangular spectrum, which leads to the necessity of guard band between WDM channels. To overcome these limitations, another technique based on phase-locked flat-comb generation was demonstrated to obtain sinc-shaped pulse trains [5]. The proofof-principle setup was based on intensity modulators driven by radio-frequency (RF) tones. This simple setup is cost-effective, but the generated bandwidth remains limited to three times the electronic bandwidth of the modulators.

In order to overcome the bandwidth limitation, we show that a nonlinear stage based on Kerr effect in highly nonlinear fiber (HNLF) can be used to expand the bandwidth of the generated comb while maintaining the high flexibility and reconfigurability advantages of the original technique. The proposed principle for the Nyquist sinc-shaped pulse train generation is sketched in Fig. 1 . Three stages of rectangular-shaped in-phase optical frequency comb generators (RI-OFCG's) are used to achieve a Nyquist-sinc pulse train from an initial optical continuous wave (CW) [Fig. 1(a)]. The first and third stages are based on intensity modulation, while the second stage is based on four-wave mixing (FWM) [ㅁ, 7] combined with a wavelength selective switch (WSS) [ $]$ ].

At the first RI-OFCG stage, the CW input is modulated via an intensity modulator $\left(\mathrm{IM}_{1}\right)$, with a sinusoidal wave. For a final desired repetition rate $f_{R}$, this sinusoidal wave frequency is set at $N \times f_{R}$, where $N$ is the number of optical tones generated at the 3rd RI-OFCG stage for a single optical carrier at its input. The amplitude of the carrier and first-order tones are equalized, while higher order tones are strongly attenuated by carefully setting the amplitude and bias voltages of $\mathrm{IM}_{1}$ [Fig. 1(b)].

The modulated signal is amplified by an EDFA and sent into a cascade of HNLF. Through FWM, energy is transferred from the three main spectral lines to new spectral components at a multiple of $N \times f_{R}$. The number of spectral lines and their amplitudes at the output of the 2nd RIOFCG stage depend on the initial power spectral density (PSD) of the three initial spectral lines, the power sent into the HNLFs, as well as the dispersion properties of the fibers used. Hence, by adjusting the EDFA output power and dispersion engineering of the fiber link [6], the number of lines with minimized PSD variation can be optimized. While the maximum power at the HNLF input is limited by stimulated Brillouin scattering (SBS), the SBS threshold of the first HNLF can be increased by up to $5 \mathrm{~dB}$ when the three initial components have equal PSD. Using different spans of HNLFs further exalts FWM while avoiding SBS [9]. The generated comb is then filtered by a WSS in order to select $K$ of its spectral components. The desired filter is programmed to equalize the amplitude and adjust the phases of the selected components. This results in a flat comb of $K$ equally spaced in-phase components with a $N \times f_{R}$ spacing [Fig. 1 $\underline{\text { (c) }]}$ 
Lastly, the optical frequency comb goes to the 3rd RIOFCG stage where it is re-modulated in an intensity modulator $\left(\mathrm{IM}_{2}\right)$. If a single $\mathrm{RF}$ tone is applied, each component of the comb will generate two first-order sidebands, thus expanding the number of lines within the comb. Further scaling can be achieved by multiplexing $\mathrm{RF}$ tones in the electrical domain. Once again, by adjusting the bias and RF power in order to emphasize the generation of the first-order sidebands, a flat comb with minimized outer sidebands can be obtained. In this way, the 3rd RI-OFCG stage allows to down rate the repetition frequency of the sinc-shaped pulse train to the frequency $f_{R}$, while maintaining the square shape of the optical comb.

Note that the use of IMs-based RI-OFCG at the 3rd stage requires setting the same state of polarization (SOP) for all the input spectral components. Such setting is difficult to reach with a polarization controller (PC) based on wave plate because of its inherent wavelengthsensitivity. To overcome such restriction, the PC is placed at the input of the 2nd RI-OFCG stage. Since components generated by FWM possess the same SOP as the initial components and the PMDs of the HNLFs and pigtails are negligible, alignment is preserved at $\mathrm{IM}_{2}$ input.

In order to experimentally illustrate the flexibility of the proposed scheme in terms of pulse duration, as well as repetition rate, three specific cases were investigated. For all cases, intensity modulators had a 40-GHz electrical bandwidth, while the FWM process was optimized to achieve efficient bandwidth expansion to at least nine times the initial modulation. To that end, the 2nd RI-OFCG stage was composed of two HNLFs: $\mathrm{HNLF}_{1}$ is $350 \mathrm{~m}$ long with zero dispersion wavelengths (ZDW) at $1569 \mathrm{~nm}$, while $\mathrm{HNLF}_{2}$ is $500 \mathrm{~m}$ long with ZDW at $1553 \mathrm{~nm}$. The

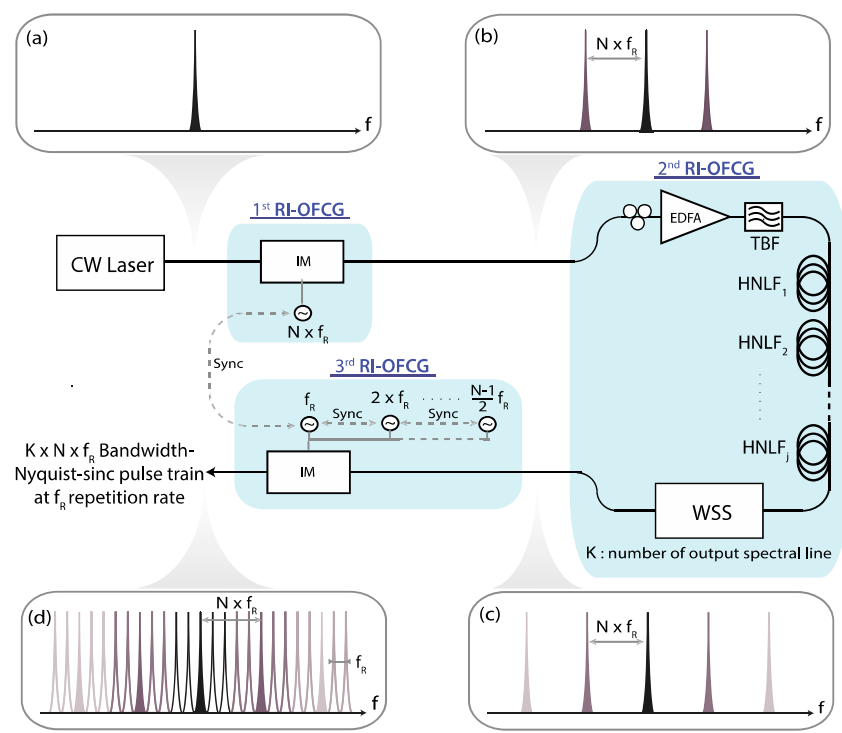

Fig. 1. Nyquist sinc-shaped pulse train generation: (a) input spectral line; (b) optical comb at the first RI-OFCG output; (c) optical comb at the second RI-OFCG output; (d) optical comb at the third RI-OFCG output; RI-OFCG, rectangularshaped in-phase optical frequency comb generator; WSS, wavelength selective switch. $f_{R}$, repetition rate; $K$, number of spectral lines at the 2nd RI-OFCG output; N, number of spectral lines generated by the 3rd RI-OFCG for each input spectral line (illustrated for odd $\mathrm{N}$ ).
$\mathrm{CW}$ laser wavelength was tuned at $1557 \mathrm{~nm}$ in order to be close to both ZDW of the HNLFs.

First, a 10-GHz repetition rate Nyquist sinc-shaped pulse train source was experimentally implemented. At the 1st RI-OFCG stage, the intensity modulator $\mathrm{IM}_{1}$ was driven with a 30-GHz clock in order to obtain three spectral lines separated by 3 times the desired $f_{R}$ [Fig. 2(a)]. Amplitude and bias voltages of $\mathrm{IM}_{1}$, as well as the output power of the EDFA, were optimized in order to have 9 equally spaced components within a 6-dB PSD variation at the output of $\mathrm{HNLF}_{2}$. At this point, a $30-\mathrm{GHz}$ pulse train is synthesized. Figure 2(b) shows the obtained spectrum. Under these conditions, the spectrum at the output of $\mathrm{IM}_{1}$ exhibited almost equal PSD components. The optimized power at the input of $\mathrm{HNLF}_{1}$ was $22.5 \mathrm{dBm}$, which is $5 \mathrm{~dB}$ above the SBS threshold of that fiber for monochromatic light.

Using the WSS, a desired number of central spectral line was selected, their amplitudes were equalized, and the spectral components put in phase. Figures 3(a), 3(b), and 3(c) show the obtained spectrum with bandwidth of (a) $1 \overline{50 \mathrm{GHz}}$, (b) $210 \mathrm{GHz}$, and (c) $270 \mathrm{GHz}$ obtained for 5 , 7 , and 9 selected spectral lines, respectively. The corresponding temporal profiles are respectively depicted in Figs. 3(d) $-3(\mathrm{f})$. More than $40-\mathrm{dB}$ rejection of the outer band spectral components was measured, restricted by the noise floor of the OSA. The temporal waveforms recorded on the optical-sampling oscilloscope (OSO) show sinc-shaped pulse trains at $30 \mathrm{GHz}$ repetition rate. Depending on the number of spectral lines, pulses with peak-to-first-zero crossing of $6.7,4.7$, and $3.7 \mathrm{ps}$ are obtained, in accordance with the properties of the sinc function.

The 30-GHz repetition rate Nyquist sinc-shaped pulse train was sent to the last RI-OFCG stage, which consisted of $\mathrm{IM}_{2}$ driven by a 10-GHz clock. By optimizing the modulator bias and amplitude voltage, 3 equal amplitude components with $10 \mathrm{GHz}$ spacing were generated from each of the initial spectral components with minimized higher order sidebands. Rectangular frequency combs still spanning 150,210 , or $270 \mathrm{GHz}$ were obtained but with a number of spectral lines increased to 15,21 , and 27, respectively, as depicted in Figs. $\underline{4(\mathrm{a})}-\underline{4(\mathrm{c})}$.

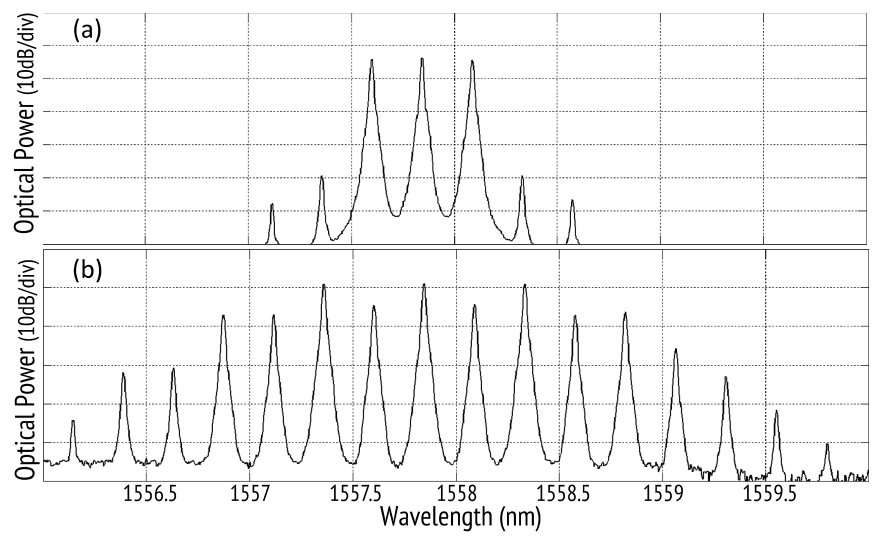

Fig. 2. Measured spectra after (a) the first RI-OFCG stage and (b) the HNLFs at the second RI-OFCG for the generation of Nyquist sinc-shaped pulse train at $30 \mathrm{GHz}$ repetition rate. 


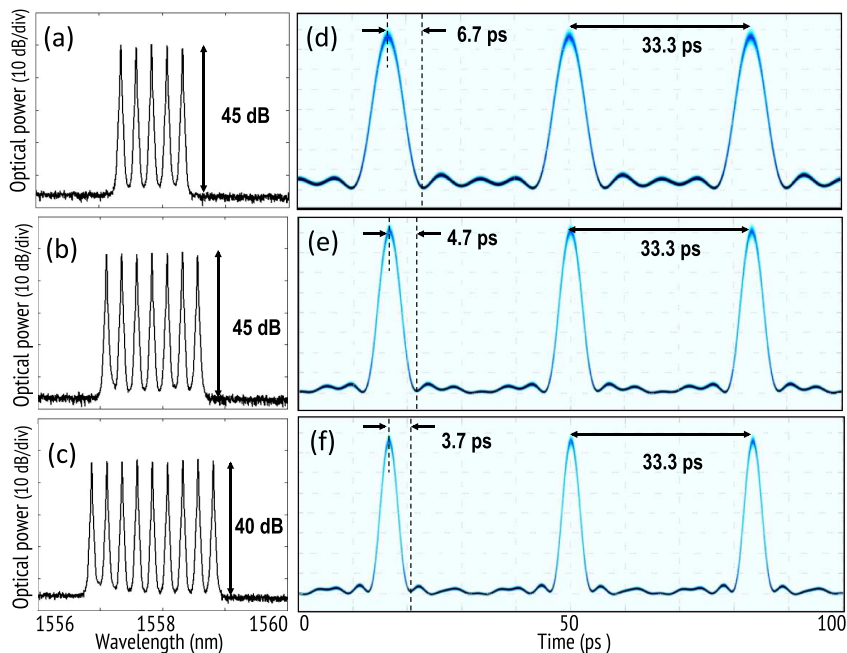

Fig. 3. Nyquist sinc-shaped pulse train at 30-GHz repetition rate (with $N=1$ ). Measured spectrum for a bandwidth of (a) $150 \mathrm{GHz}$, (b) $210 \mathrm{GHz}$, and (c) $270 \mathrm{GHz}$ (resolution $0.01 \mathrm{~nm}$ ), and the corresponding temporal profiles in (d)-(f).

More than $32.5 \mathrm{~dB}$ rejection of out-of-band components was achieved. The corresponding measured temporal profiles [Figs. 4(d)-4(f)] are sinc-shaped pulse train at $10 \mathrm{GHz}$ repetition rate. The pulse duration, which is related to the bandwidth of the spectrum, remained identical as for the previous results at $30 \mathrm{GHz}$.

Secondly, a 40-GHz repetition rate Nyquist sinc-shaped pulse train source was also experimentally implemented. Compared to the first case, only the two first RI-OFCGs were used $(N=1)$, and the clock frequency driving $\mathrm{IM}_{1}$ was set to $40 \mathrm{GHz}$ in order to reach the desired repetition rate. Figures $\underline{5(\mathrm{a})}$ and $\underline{5(\mathrm{~b})}$ depict the measured $360-\mathrm{GHz}$ bandwidth rectangular spectrum comb obtained for $K=$ 9 spectral lines, and the associated temporal profile, respectively.

In this case the flat square spectrum comb-less than $1 \mathrm{~dB}$ ripple for more than $50 \mathrm{~dB}$ rejection of the outer

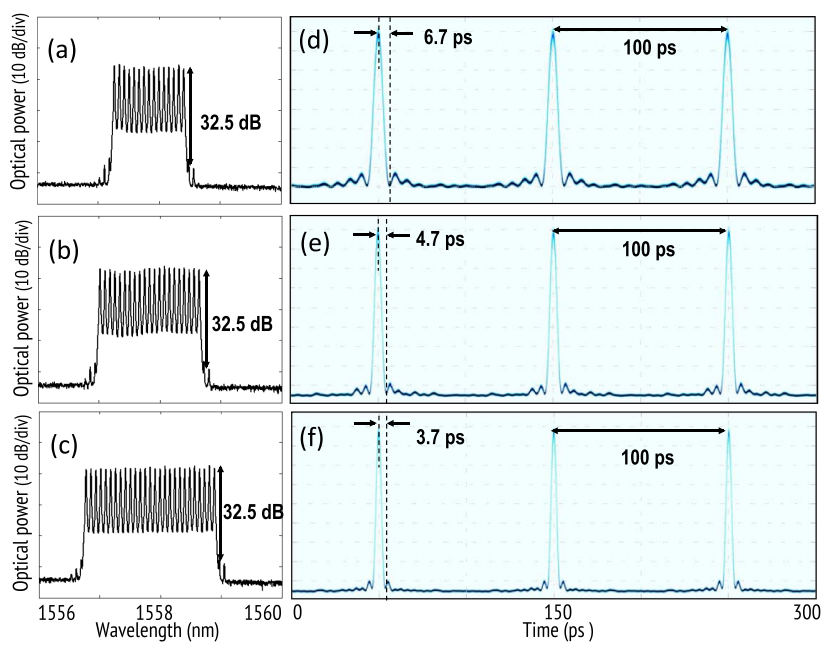

Fig. 4. Nyquist sinc-shaped pulse train at $10-\mathrm{GHz}$ repetition rate (with $N=3$ ). Measured spectrum for a bandwidth of (a) $150 \mathrm{GHz}$, (b) $210 \mathrm{GHz}$, and (c) $270 \mathrm{GHz}$ (resolution $0.01 \mathrm{~nm}$ ), and the corresponding temporal profiles in (d)-(f).

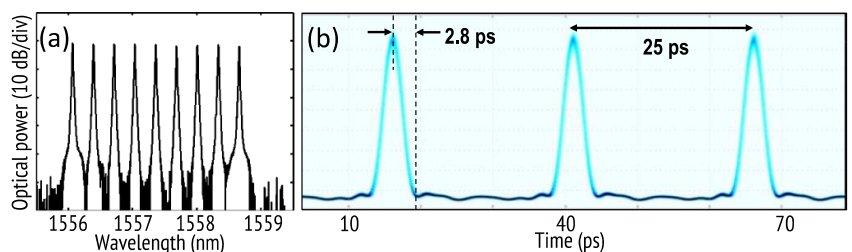

Fig. 5. Nyquist sinc-shaped pulse train at $40-\mathrm{GHz}$ repetition rate. (a) Measured spectrum for a bandwidth of $360 \mathrm{GHz}$ (resolution $0.01 \mathrm{~nm}$ ) and (b) the corresponding temporal profile.

band spectral component-leads to a shorter Nyquist sinc-shaped pulse with 2.8-ps peak-to-zero crossing. A residual linewidth broadening scaled with the order of spectral lines was generated by FWM $[10,11]$. The reduction of the optical signal-to-noise ratio (OSNR) for newly generated spectral lines is due to imperfect phase correlation of the FWM components, here intensified by increase of line frequency spacing [11]. In contrast to the result obtained in [2], where the repetition rate is fixed by the mode-locked laser, our proposed scheme allows a full flexibility in term of repetition rate up to the limit of the electrical bandwidth of the IM in the 1st RI-OFCG stage. Nevertheless, decreasing the repetition rate worsens the rejection of the outer band spectral components due to the limited 3.5- $\mathrm{dB} / \mathrm{GHz}$ attenuation slope of the WSS. As a consequence, for a minimum $21 \mathrm{~dB}$ rejection of outer band spectral components [5], the repetition rate cannot be set below $6 \mathrm{GHz}$. Moreover, the frequency accuracy of the WSS is about $5 \mathrm{GHz}$. Hence, for lower repetition rates the 3rd RI-OFCG has to be used.

Finally, a $5 \mathrm{GHz}$ repetition rate Nyquist sinc-shaped pulse train source was experimentally realized. The intensity modulator $\mathrm{IM}_{2}$ of the 3rd RI-OFCG stage was simultaneously driven by two RF clock generators, at 5 and $10 \mathrm{GHz}$, giving in such way a frequency spacing equal to the desired repetition rate, $f_{R}=5 \mathrm{GHz}$. In this configuration, to generate a comb with a spectral spacing equal to $N=5$ times $f_{R}$ at the 1st RI-OFCG stage, a 25GHz clock was applied to $\mathrm{IM}_{1}$. At the 2nd RI-OFCG stage, a $K=9$ spectral line square comb was chosen and injected in the 3rd RI-OFCG stage. Then at the last stage, the phase synchronization and amplitude equalization of the 5 tones generated for each $K=9$ initial spectral line was performed by fine bias and amplitude voltage setting on the $5-\mathrm{GHz}$ and $10-\mathrm{GHz}$ clocks.

Figure 6(a) depicts the measured spectrum for 45 spectral lines covering a 225-GHz bandwidth, and Fig. 6(b) shows the corresponding temporal profile. A $28-\mathrm{dB}$ rejection of the outer band spectral components was measured. This higher level of rejection, compared to the

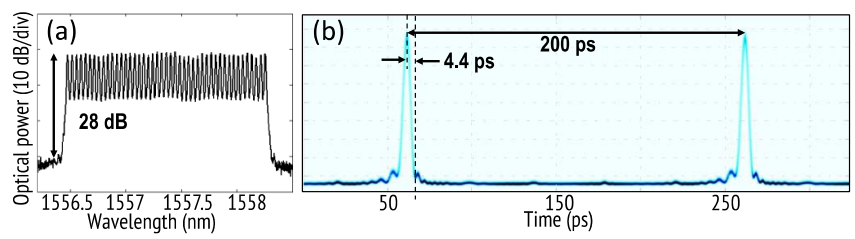

Fig. 6. Nyquist sinc-shaped pulse train at 25-GHz repetition rate (with $N=5$ ). (a) Measured spectrum of the $225-\mathrm{GHz}$ bandwidth rectangular frequency comb (resolution $0.01 \mathrm{~nm}$ ), (b) corresponding temporal profile. 


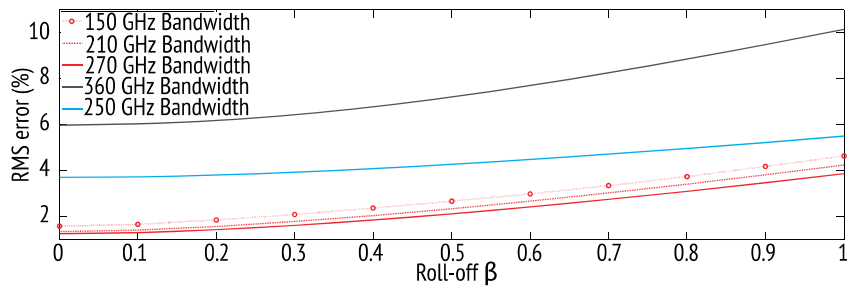

Fig. 7. RMS error between the theoretical sinc and measurement of Nyquist sinc-shaped for a bandwidth of $150-\mathrm{GHz}$ (red line), $210-\mathrm{GHz}$ (red dash), 270-GHz (red dot) at 10-GHz repetition rate, and for a bandwidth of $225 \mathrm{GHz}$ (blue) and $360 \mathrm{GHz}$ (dark) at, respectively, 5- and 40-GHz repetition rate.

$17 \mathrm{~dB}$ feasible by the WSS over $5 \mathrm{GHz}$, is obtained thanks to the minimized higher order sidebands generated via $\mathrm{IM}_{2}$. Thus, the square shape of the comb spectrum is maintained for low repetition rate $(<6 \mathrm{GHz})$. The temporal pulse train recorded was sinc-shaped at $5 \mathrm{GHz}$ repetition rate with 4.4-ps peak-to-zero width at FWHM, in agreement with theory. Some small residual peaks are observed, which are due to slight phase mismatch and unequal level of the resulting 45 spectral lines.

The quality of the measured Nyquist sinc-shaped pulses were analyzed by comparing them with the theoretical expression of the Nyquist-sinc pulse $r(t)$ as a function of the roll-off factor $\beta$, as described in Eq. (1) []]:

$$
r(t)=\frac{\sin \left(\frac{2 \pi t}{\tau_{p}}\right)}{\frac{2 \pi t}{\tau_{p}}} \cdot \frac{\cos \left(\frac{2 \pi \beta t}{\tau_{p}}\right)}{1-\left(\frac{4 \beta t}{\tau_{p}}\right)^{2}},
$$

where $\tau_{p}=2 /\left(N \times K \times f_{R}\right)$ is the pulse duration between zero crossing.

Figure 7 shows the root mean square (RMS) error between the experimental Nyquist pulses compared to the theoretical Nyquist-sinc function for a 150, 210, $270 \mathrm{GHz}$ bandwidth at $10-\mathrm{GHz}$ repetition rate, as well as for a 225and $360-\mathrm{GHz}$ bandwidth at the respective $5-\mathrm{GHz}$ and $40-$ $\mathrm{GHz}$ repetition rate. Minimum RMS error is reached when the $\beta$ is zero. Such result is in agreement with the flatness ( $<1 \mathrm{~dB}$ for all cases) of the frequency combs and the level of out-of-band rejection ratio (a least $28 \mathrm{~dB}$ ) obtained. However, up to $6 \%$ of RMS error is obtained for the $360-\mathrm{GHz}$ comb. The obtained deviation comes from slight deviation in the phase locking of the various spectral components (similar to the $225-\mathrm{GHz}$ case) but also from the limitation imposed by the $500-\mathrm{GHz}$ bandwidth of the OSO, which did not allow rigorous detection of the zero crossing point.

We proposed an extension of the scheme presented in $[\underline{5}, 6]$, used to generate roll-off free sinc-shaped pulse trains with 2 stages of RI-OFCG based on intensity modulation. An additional RI-OFCG stage, based on FWM in multiple spans of HNLF combined with a WSS, was added between the 2 existing RI-OFCG's stages. This nonlinear-optic stage was designed to generate equalized in-phase spectral lines beyond the electronic bandwidth of electro-optical devices. This was key for generating all-optical Nyquist-sinc shaped pulses with pulse duration divided by up to 3 times at any repetition rate. In addition to the ability of changing the duration of the Nyquist-sinc shaped pulses owing to the WSS, the method allows for full tunability of the repetition rate in the limit of the electrical bandwidth of the intensity modulators. Close to zero roll off coefficients were demonstrated for ultrashort pulses at three different repetition rates, namely 5, 10, and $40 \mathrm{GHz}$. The presented scheme can be used to control the way that the total bandwidth is shared among users in granularity flexible and ISI-free O-TDM networks. The bandwidth expansion can be easily upgraded, for instance by using more spans of shorter HNLFs or by adding a phase modulator between the 1st and 2nd RI-OFCG, to include more spectral components and hence shorter sinc pulses, which can be of particular interest for other photonic assisted technologies, such as photonic analog-to-digital conversion or optical sampling. Using properly designed passive optical filters and voltage-controlled oscillators (VCOs), the cost could be drastically reduced. It is also noteworthy that costeffective implementation using photonics integrated circuits could be done for dedicated repetition rate and bandwidth.

The authors acknowledge T. Sylvestre from Femto-ST institute and Sumitomo Electric for providing the two HNLFs and L. Thévenaz from École polytechnique Fédérale de Lausanne (EPFL) for fruitful discussions. This work was supported by the Hasler Foundation (project number 14011).

\section{References}

1. R. Schmogrow, M. Winter, M. Meyer, D. Hillerkuss, S. Wolf, B. Baeuerle, A. Ludwig, B. Nebendahl, S. Ben-Ezra, J. Meyer, M. Dreschmann, M. Huebner, J. Becker, C. Koos, W. Freude, and J. Leuthold, Opt. Express 20, 317 (2012).

2. M. Nakazawa, H. Toshihiko, R. Peng, and G. Pengyu, Opt. Express 20, 1129 (2012).

3. A. Vedadi, M. A. Shoaie, and C.-S. Brès, Opt. Express 20, 27344 (2012).

4. M. A. Shoaie, A. Mohajerin-Ariaei, A. Vedadi, and C.-S. Brès, Opt. Express 22, 4606 (2014).

5. X. Zhou, X. Zheng, H. Wen, H. Zhang, Y. Guo, and B. Zhou, Opt. Commun. 284, 5284 (2011)

6. M. A. Soto, M. Alem, M. A. Shoaie, A. Vedadi, C.-S. Brès, L. Thévenaz, and T. Schneider, Nat. Commun. 4, 31347 (2013).

7. G. A. Sefler and K. Kitayama, J. Lightwave Technol. 16, 1596 (1998).

8. G. Baxter, S. Frisken, D. Abakoumov, H. Zhou, I. Clarke, A. Bartos, and S. Poole, in Proceedings of the Optical Fiber Communication Conference (Optical Society of America, 2006), paper OTuF2.

9. X. P. Mao, R. W. Tkach, A. R. Chraplyvy, R. M. Jopson, and R. M. Derosier, IEEE Photon. Technol. Lett. 4, 66 (1992).

10. J. Zhou, R. Hui, and N. Caponio, IEEE Photon. Technol. Lett. 6, 434 (1994).

11. Z. Tong, A. O. J. Wiberg, E. Myslivets, B. P. P. Kuo, N. Alic, and S. Radic, Opt. Express 20, 17610 (2012). 